\title{
Performance of Ultra-Violet Laser Pulsing with a Wire-Geometry, Moderately Focused Atom Probe System (EIKOS)
}

Robert M. Ulfig ${ }^{1}$, Joseph Bunton ${ }^{1}$, Dan Lenz ${ }^{1}$, Yimeng Chen ${ }^{1}$, Katherine P. Rice ${ }^{1}$, Ty J. Prosa ${ }^{1}$ Peter. H. Clifton ${ }^{1}$ and David J. Larson ${ }^{1}$

1. CAMECA Instruments Inc., 5470 Nobel Drive, Madison, WI 53711 USA.

* Corresponding author: Robert.Ulfig@ ametek.com

Atom probe tomography has been used for decades to obtain nanoscale chemical information on a variety of materials. Hardware developments in recent years have increased the fraction (field of view) and total number (depth) of ions detected, improved specimen yield, and allowed for a wider range of material systems to be investigated [1]. In 2016, CAMECA ${ }^{\circledR}$ introduced a lower cost of ownership atom probe. The EIKOS ${ }^{\mathrm{TM}}$, with a simplified design and a smaller footprint than the LEAP 5000 ${ }^{\mathrm{TM}}$ (Local Electrode Atom Probe) makes it possible for a wider range of research institutions to include atom probe tomography as a routine part of their materials characterization process [2].

The initial implementation of EIKOS utilized a fiber-based, $532 \mathrm{~nm}$ (green), $1 \mathrm{~ns}$ pulse-duration laser system to provide simplicity of design, construction, and use. Previous work with LEAP ${ }^{\circledR}$ systems demonstrated the improved spectral quality and yield achieved with shorter wavelength laser pulsing [3]. The EIKOS platform was designed to enable the integration of different laser sources and recently UVlaser systems that meet power, beam quality, and form factor requirements for EIKOS have become available. In this study a $355 \mathrm{~nm}$ (UV) $\sim 1 \mathrm{~ns}$ pulse-duration fiber-based laser source was configured for use with the EIKOS system and the mass spectral performance is compared to data that had been previously collected with the green laser system for several material systems.

Aluminum, stainless-steel, silicon, gallium nitride, and geological materials were prepared for atom probe tomography using standard methods [4,5]. Analysis conditions (electric field, ion flux, temperature, etc.) were chosen to match the data collected with green laser-pulsing. Mass resolving power improvements ranged from a modest $15 \%$ in aluminum samples to over $100 \%$ for silicon-based samples (see Fig. 1). Sensitivity to low-concentration dopants in silicon was also demonstrated to be significantly improved (up to 50\% using the LDL method see Fig. 1d) [6]. Geological materials have not been quantitatively studied using green laser systems. In this paper Zircon crystals (obtained from the Beartooth Mountains and previously used to date geological features) were studied and the results showed homogeneous Zircon with a nominally stoichiometric analysis, $\mathrm{ZrSiO}_{4}$ (Fig. 2) [7]. A moderately-focused UV-laser in the EIKOS has demonstrated significant improvements in mass resolving power and detection sensitivities. The impact of yield improvement and the potential expansion of application space will also be discussed.

References:

[1] D.J. Larson et al., "Local Electrode Atom Probe Tomography”, (Springer, New York) (2013).

[2] D.J. Larson et al., JOM 70 (2018), p. 1776.

[3] J. Bunton, et al., Microscopy and Microanalysis 13 (2007), p. 418.

[4] A.J. Melmed, J.V.S.T. B9 (1991), p. 601.

[5] K. Thompson et al., Ultramicroscopy 107 (2007), p. 131.

[6] L.A. Currie, Analytical Chemistry 40 (1968), p. 586. 
[7] D.A. Reinhard et al., Microscopy and Microanalysis 21 (2015), p. 851.
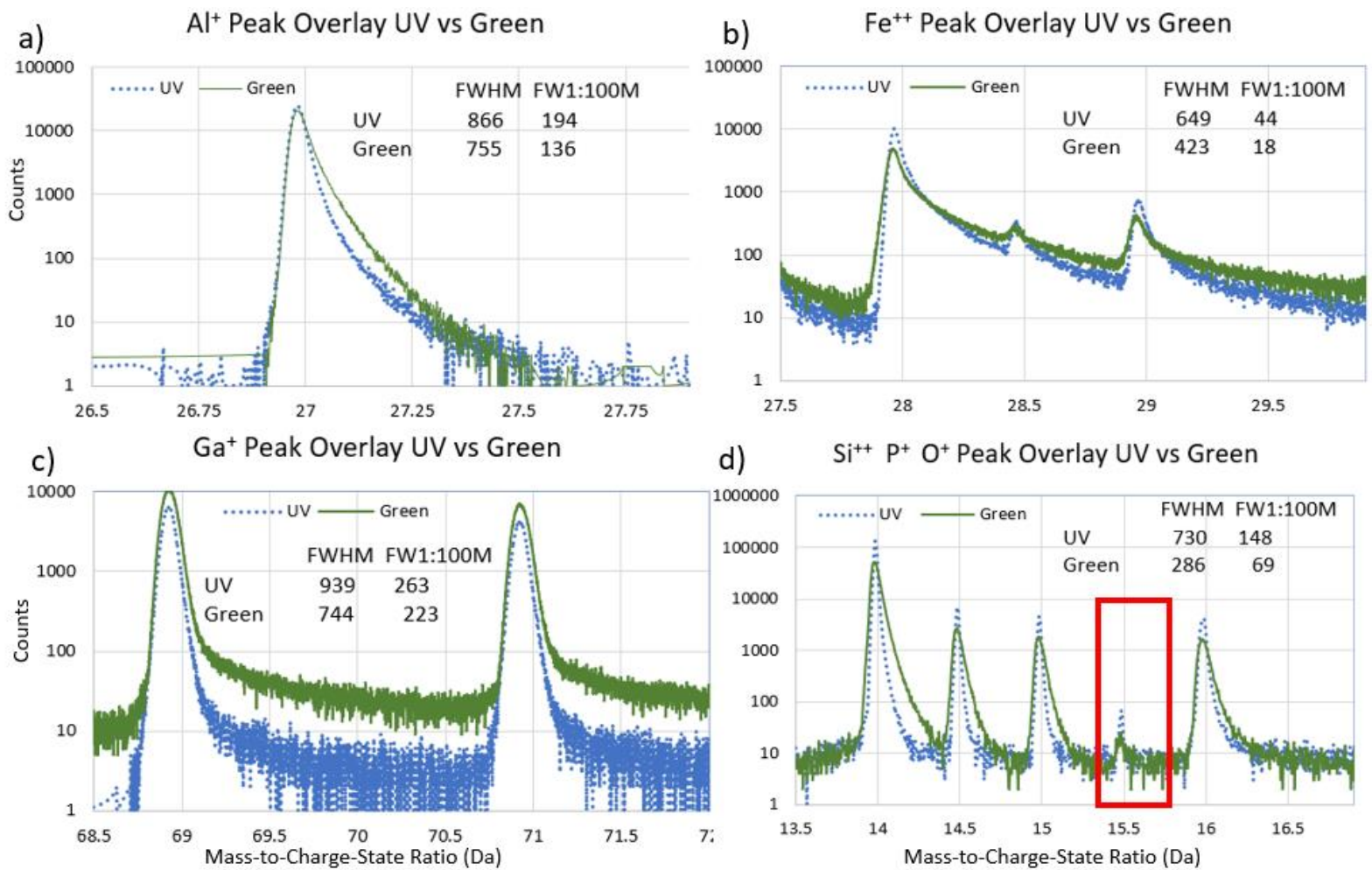

d) $\mathrm{Si}^{++} \mathrm{P}^{+} \mathrm{O}^{+}$Peak Overlay UV vs Green

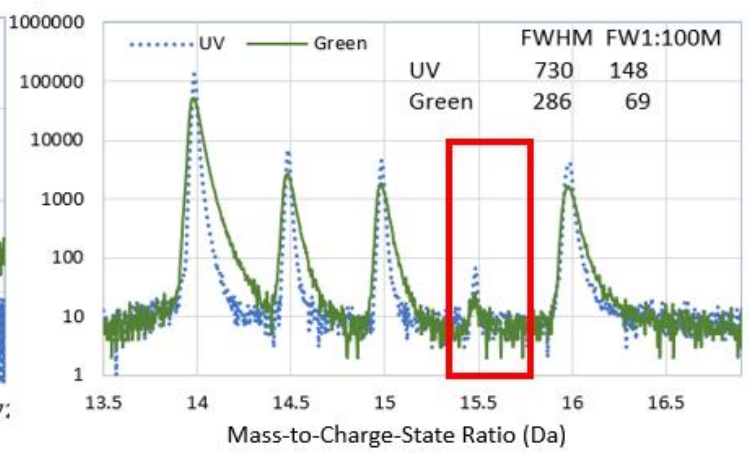

Figure 1. Superimposed log-scale mass spectra for materials analyzed with both the UV and green laser systems on EIKOS: a) Aluminum, b) Iron, c) $\mathrm{GaN}$, d) $\mathrm{Si} / \mathrm{SiO}_{2} / \mathrm{Si}$ 'yield' sample. In all tested materials, the mass resolving power improved from $15 \%$ to $>100 \%$ for the $\mathrm{SiO}_{2}$ yield sample in d). Note in d) the improved ability to identify and quantify the phosphorus dopant in the implanted poly-Si on silicon oxide sample (red box).
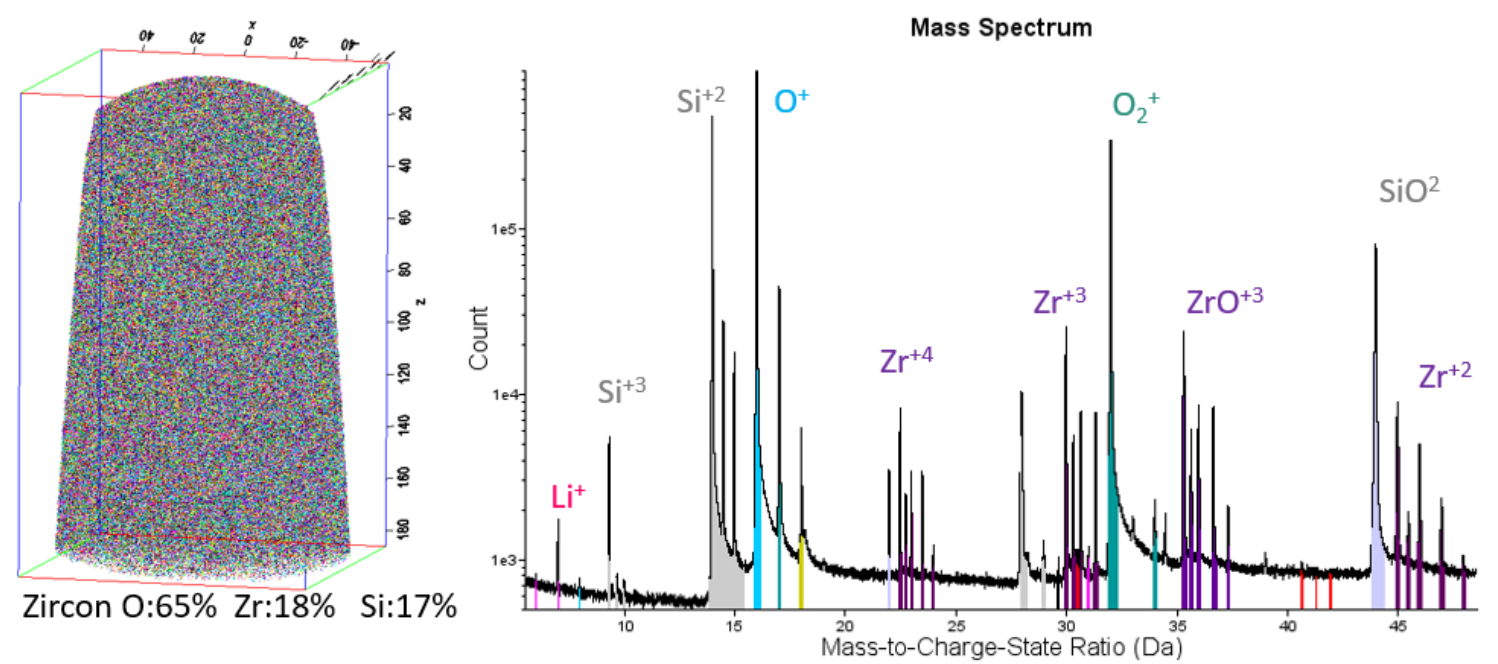

Figure 2. Three-dimensional reconstruction of a Beartooth Zircon crystal analyzed with the UV-EIKOS system: (left) atom map and (right) mass spectrum. The homogenous region of this crystal was measured with nominal stoichiometry for $\mathrm{ZrSiO}_{4}$ not previously reported with green laser systems. 